\title{
David Ragghianti: Portland. Caipira Records A60113, 2015.
}

In un articolo pubblicato nel settembre 2015 su Rock.it e dedicato ai "Dieci nuovi cantautori italiani da tenere d'occhio", il nome di David Ragghianti risulta come primo in lista, seguito da altri nomi di cantautori emergenti come Carmine Torchia, Federico Cimini, Alberto Gesù, Bombay, Frisino o Caso. Come motivazione di tale scelta si legge, tra le altre cose, che l'album d'esordio del giovane cantautore toscano, Portland (uscito proprio nel settembre 2015), sarebbe insieme "impetuoso, scuro, vellutato, poetico. Da ascoltare" ${ }^{\text {. Effettivamente }}$ le qualità che saltano all'occhio ascoltando le nove canzoni di cui Portland è composto, sono sia il carattere altamente poetico dei testi, sia la piega a volte malinconica, a volte nettamente cupa che i testi prendono a livello dei significati, sia ancora l'intensità appassionata (forse più che 'impetuosità') che emana dall'interpretazione musicale e verbale della maggior parte dei brani. Tale intensità si manifesta praticamente in tutte le canzoni in cui Ragghianti, con la sua bella voce baritonale, adotta il tipico stile cantautoriale con un'interpretazione dei testi tra cantata e parlata e un accompagnamento basato principalmente sulla chitarra acustica con pochi altri strumenti (pianoforte, percussioni, batteria), il tutto congiunto in arrangiamenti molto professionali e validi (ad opera di Giuliano Dottori) che contribuiscono molto anch'essi al suddetto effetto di 'intensità appassionata'. Questa tende però a dileguarsi dove si sperimantano altri generi musicali, e sia detto subito che ciò succede soprattutto nel sesto brano "Se non ti ammali mai" i cui ritmi di reggae semplici e un po' banali finiscono per togliere profondità anche alle parole - già di per sé poco concrete - della canzone.

Questa ci sembra comunque l'unica eccezione in un album di alta qualità che elabora poeticamente le riflessioni, i punti di vista e i dubbi di un "io" appartenente alla generazione giovane d'oggi. Il brano iniziale, "I prati che cercavo", assume un tono nettamente intimistico e ci porta dentro ambienti malinconici che sembrano fuorusciti dai ricordi ma anche, in parte, dalle fiabe. Le parole molto poetiche di questa canzone richiamano alla mente una frase espressa nei brevi cenni biografici che si trovano sul sito del cantautore: "Ho passato l'infanzia in campagna a piedi scalzi." Infatti i versi della canzone conducono in un universo campestre e autunnale fatto di prati, fiori, frutti maturi e nespole appassite, un mondo dalle reminiscenze quasi pascoliane dove non manca nemmeno l'accenno implicito (ma ricorrente perché fa parte di un ritornello) ad una morte incombente - si vedano i versi "Il comune abisso a tutti / da dove viene il nuovo / riguarda i fiori / ed è fedele alla terra". Ma il carattere fresco e nuovo di questo tema, alquanto consunto, del continuo rinnovarsi della natura tramite l'alternarsi di vita e morte, risulta proprio dal modo in cui i versi appena citati proseguono, cioè con il ricorso a delle metafore spaziali o addirittura geometriche che traspongono l'idea del ciclo naturale di vita e morte su un livello astratto, teorico, dai significati in parte enigmatici: 
[Il comune abisso a tutti ...]

s'addensa di un sapore pieno

per farsi duplice

si rappresenta

c'è un mondo immaginato

ed uno di maiuscole

un frutto gigantesco

dove ci stanno i ritorni

Un procedimento semantico simile, ma in ordine inverso, si sottende anche a "Occhi asciutti”, il quarto brano dell'album: dai versi iniziali con le loro formulazioni figurate e tendenzialmente astratte (si veda p.es. l'inizio della seconda strofa "In quell'eterno sussurrare / che non so mai da dove arrivi / ho visto frane lungo i cigli / poi lunghi argini distesi") si arriva nell'ultima strofa allo scenario ben concreto di un universo campestre idilliaco sognato e bramato:

\author{
Voglio vivere in un posto dove sorrida la natura \\ prender la pioggia goccia a goccia [...] \\ e quell'eterno canticchiare \\ delle cicale in estate \\ ripetono va tutto bene \\ non ci dobbiamo preoccupare (bis)
}

Il mondo sognato, irrimediabilmente perduto dell'infanzia si fa avanti anche nel secondo brano dell'album, "Amsterdam", e di nuovo si tratta di un mondo ambiguo perché fatto sia di attrazioni ed entusiasmi, sia di insidie, inganni e trucchi. L'elemento che rinvia marcatamente all'universo infantile è il nome di Trilli, piccola fata sorta dai film d'animazione, in una parte secondaria nel classico Disney Le avventure di Peter Pan (1953) e come personaggio principale nell'omonimo film d'animazione Trilli del 2008. Anche l'immagine della ragazza bionda con una palla rossa fra le mani che si vede sulla copertina dell'album, richiama un po' la fatina, e in ogni caso richiama anch'essa l'universo infantile. La fata che riesce a sanare tutto ciò che non funziona e ad abbellire le cose brutte tramite la sua "polvere di stelle", in "Amsterdam" viene evocata come soccorritrice che ci vorrebbe nei momenti difficili, dato che "lei non distrugge niente". Quanto ai momenti difficili di cui è questione, sembrano riferirsi proprio a quella fase di vita che separa l'infanzia dall'età adulta, in cui si rischia di "essere portato via dalle occasioni" o di "essere portato via senza impressioni" e di ritrovarsi senza il "talento" di una volta. La difficoltà di (ri)trovare un equilibrio viene descritta nel ritornello con delle immagini che variano il concetto di sospensione tra due stati: "tra terra e cielo", "tra vuoto e pieno", tra "una forza che ti spinge avanti / una forza che ti tira indietro". Ciò che resta enigmatico è invece il titolo della canzone, "Amsterdam", per il quale non si trova nessuna spiegazione nel testo o nella musica - come è ugualmente il caso, tra l'altro, per il titolo dell'intero album, Portland. Si potrebbe dire tutt'al più che questi 
nomi geografici suggeriscono un desiderio di viaggio e di lontananza, il quale sembra però essere in antagonismo con il carattere introspettivo proprio alla maggior parte delle canzoni.

Tale carattere lo condividono anche i due brani che trattano rapporti amorosi o, per dirlo in modo più neutrale, rapporti tra l'io narrativo e un "tu" femminile: "Pause estive" e "Dove conduci". L'ultimo si presenta di primo acchito come vera e propria canzone d'amore, sia con il suo andamento melodico sommessamente sereno, sia con le sue parole edificate innanzi tutto sulle immagini di una corporeità sensuale; però proprio queste immagini conducono man mano in sfere semantiche estranee e, di nuovo, ambivalenti. Se i concetti quasi simmetrici de "il mio corpo" e "[la tua] carne", "la mia fame" e "La rabbia che si fa sete" suggeriscono un rapporto carnale di mutua sintonia, il concetto del dolore fisico che percorre l'intera canzone racconta una storia diversa, una storia di ferite e piaghe che sfocia in "una ricerca ispirata e precisa / dove il dolore decida" - cioè, in fin dei conti, in una separazione. Letta dalla sua fine, questa canzone si rivela essere perciò la storia di un amore già finito o, addirittura, già superato visto che "io non avevo mai pensato che / fosse possibile vivere senza te". Un atteggiamento ancora più disincantato (ma senza essere pessimistico, piuttosto serenamente rassegnato), regna in "Pause estive", storia di un rapporto che non si è mai veramente avviato dopo che il primo contatto si è concluso con un sorriso impacciato ("non sapevi più che fare") da parte di lei. In un rapporto con prospettiva di lieto fine, invece, non dovrebbe essere così: l'io narrativo congettura che "quando tutto funziona funziona da sé" e ne conclude che è meglio non insistere, ma rimandare il possibile rapporto, senza rimpianto o bisogno di sentirsi offesi, ad un futuro indefinito ("niente di personale nel dirsi di no").

In un modo molto più astratto è questione di rapporti anche in "Tema del filo". L'immagine del filo non vi rappresenta soltanto il tema centrale, come suggerisce il titolo, ma diventa anche una metafora efficace per i vari tipi di "legami", di "nodi" e di "strappi" che determinano le nostre vite: dai legami interpersonali ("Hai bisogno di un filo / [...] per tenere e lasciare / per mettere insieme") attraverso il continuo rischio che corriamo di impigliarci nella rete delle proprie illusioni o dei propri errori ("Prima ci si arrotola a vortice / e poi si corre a lungo / fin dove arriva il fiato") fino ai fili invisibili che collegano il nostro vissuto diurno con quello notturno dei sogni ("Hai bisogno di un filo / [...] per lanciare l'esca di giorno / poi la notte tirare su un sogno"). Il sogno che, insieme ai ricordi, rappresenta una delle tematiche centrali dell'intero album, si identifica in questa canzone addirittura con il dono di far dissolvere in aria i problemi che risultano dal nostro "arrotolarsi a vortice" - questo almeno lo sembra suggerire il verso del ritornello ripetuto più volte durante la canzone "al resto pensa il sonno / a ricordarci tutto". Musicalmente "Tema del filo" è una delle canzoni più riuscite, grazie ad un intrecciarsi (fedele alla metafora del filo) di generi musicali, cioè l'alternarsi del caratteristico stile cantautoriale con delle dolci sonorità latinoamericane nel ritornello le quali in questo caso (diversamente dal reggae del seguente brano "Se non ti ammali mai", a cui abbiamo già accennato) portano a dei risultati del tutto convincenti.

L'album si conclude con due canzoni dalle melodie calme e dagli arrangiamenti poco appariscenti, ma che a livello del testo sembrano voler minare l'eccesso di discrezione e di understatement. La prima delle due, “300 anni”, canzone che per il modo in cui è cantata 
gorgoglia come un piccolo ruscello che si nota appena, tradisce però dei grandi propositi sia con l'universalismo del suo punto di vista che vaga tra il fondo del mare e i silenzi del bosco, tra bene e male, tra brividi e peccati, sia con il coraggio del suo voler "riconquistare" la vita in "un giorno di quei tanti a caso". Un'intensità ancora maggiore, ma ben diversa per i contenuti espressi, è quella creata dalle parole del brano finale dell'album, "Raffiche di fuga". Con la lunga enumerazione di cose sentite, viste e pensate di cui il suo testo si compone, essa crea uno stato d'animo nettamente cupo che ne fa la canzone più esplicitamente pessimistica - $o$ almeno scettica - dell'album. È come se il cantautore-poeta vi volesse mettere in primo piano quanto nelle altre canzoni rimane sottinteso. È molto marcata innanzi tutto la costatazione della caducità e vanità generale della vita ("questo perpetuo camminare invano", "questo abbaglio chiamato vita", "queste tracce che portano a niente"), ma anche la visione disincantata del passato ("senza rimpianti né vie di fuga") e l'accentuazione del proprio disorientamento ("proprio niente vado a cercare"). Procura un senso di consolazione solo il ritornello, le cui parole ritraggono un paesaggio invernale con finestre illuminate sul mare, visione che conferisce un senso di intimità e suggerisce un luogo dove ci si potrebbe sentire al sicuro.

In fin dei conti, ciò che convince di più nell'album d'esordio di David Ragghianti è proprio quel suo carattere ambiguo e 'scuro', per cui sotto le suggestioni di un universo idilliaco dell'infanzia o di un rapporto amoroso felice si aprono delle crepe profonde nelle quali si intravvede un mondo instabile che rischia di sgretolarsi. Per creare questo stato di sospensione e di precarietà, ci vogliono dei procedimenti poetici appropriati che l'artista, come viene sostenuto anche in una delle recensioni su internet, riesce ad adoperare con talento e con fiuto: "[...] le qualità del Nostro vanno ricercate in quella grana di significati nascosti tra le righe, negli a capo eleganti e in accostamenti di parole mai usate a casaccio". ${ }^{3}$ I paragoni che si leggono in questa e in altre recensioni con un grande del cantautorato italiano come Ivano Fossati, ${ }^{4}$ già da questo punto di vista ci sembrano interamente giustificati. Si tratta di un cantautore da seguire, e anche il nuovo disco già uscito di Ragghianti, l'EP Archipelaghi (2016), vale sicuramente l'ascolto.

\section{Gerhild FUCHS (Innsbruck)}

\section{Note}

1 https://www.rockit.it/articolo/nuovi-cantautori-italiani-emergenti (consultazione 01.11.2016).

2 http://davidragghianti.com/about (consultazione 01.11.2016).

$3 \mathrm{http} / /$ sentireascoltare.com/recensioni/david-ragghianti-portland/ (consultazione 01.11.2016).

4 Cf. p.es. http://sentireascoltare.com/recensioni/david-ragghianti-portland/ e http://www.ondar ock.it/recensioni/2015_davidragghianti_portland.htm (consultazione 01.11.2016). 